Pacific Journal of Mathematic 


\title{
SEMILATTICES HAVING BIALGEBRAIC CONGRUENCE LATTICES
}

\author{
GarR S. Lystad AND Albert R. Stralka
}

\begin{abstract}
The congruence lattice of a semilattice is algebraic (=compactly generated). In this paper those semilattices having bialgebraic congruence lattices are characterized. We are also able to characterize those semilattices having the stronger property that their congruence lattices support a compact, Hausdorff topology which makes them into topological lattices.
\end{abstract}

Let $\mathscr{P}$ be the category of (meet) semilattices and meet-preserv ing maps. A congruence on a semilattice $S$ is a subset of $S \times S$ which is both a subsemilattice and an equivalence relation. When ordered by inclusion $\Theta(S)$, the set of all congruences on $S$, becomes a coatomically generated (i.e., every element is an infimum of coatoms), algebraic (=compactly generated) lattice (cf. [3], [8]). As such it will have very strong completeness or topological properties examplified by the fact that it supports a naturally defined topology relative to which it becomes a compact topological semilattice. In this paper we are concerned with those semilattices whose congruence lattices have even stronger topological properties. First, we are able to characterize, both externally and internally, those semilattices $S$ for which $\Theta(S)$ is bialgebraic (i.e., both $\Theta(S)$ and its dual are algebraic). The external characterization is based upon whether $S$ has either of two very elementary semilattices as a quotient.

Bialgebraic lattices will then support two naturally defined topologies. Meet is continuous relative to one and join is continuous relative to the other. In general, these two topologies do not agree. However, in many interesting cases, such as the lattice of all subsets of a set, they do. Bialgebraic lattices in which these topologies coincide will be caljed coordinated. In $\S 3$ we characterize those semilattices having coordinated bialgebraic congruence lattices. Again, both internal and external characterizations are given. For the external characterization we need only add a third familiar semilattice to the two needed for the bialgebraic situation.

The theory of algebraic lattices is fully developed in the book by Crawley and Dilworth [2] where they are called compactlygenerated lattices. Basic information about congruence lattices on semilattices is to be found in the papers of Dean and Oehmke [3] and Papert [8]. Kent and Atherton have previously discussed 
bialgebraic lattices in the papers [1] and [6].

1. Preliminaries. By a semilattice we shall always mean meetsemilattice. When we discuss join-semilattices we shall always use the modified form. For a $\mathscr{P}$-morphism $\varphi: S \rightarrow T$ we define [ $\varphi$ ] to be $\left\{\left(s_{1}, s_{2}\right) \in S \times S: \varphi\left(s_{1}\right)=\varphi\left(s_{2}\right)\right\}$. Clearly, every member of $\Theta(S)$ is of this form. An element $k$ of a lattice $L$ is compact if whenever $A$ is an upward directed subset of $L$ with $\sup A \geqq k$ there is an element $a$ of $A$ such that $a \geqq k$. The set of compact elements of $L$ will be denoted by $K(L)$. For a partially ordered set $P$, a point $x$ of $P$ and a subset $A$ of $P$, we define $\downarrow x=\{p \in P: p \leqq x\}$ and $\downarrow A=\cup$ $\{\downarrow a: a \in A\}$. The sets $\uparrow x$ and $\uparrow A$ are defined dually. A lattice $L$ is algebraic if it is complete and for each $x \in L, x=\sup (\downarrow x \cap K(L))$. The terms cocompact and coalgebraic have the expected meaning. $L$ is coatomically generated if each element $x$ of $L$ is the infimum of the set of all coatoms of $L$ contained in $\uparrow x$.

For a semilattice $S$, the zero, or least element of $\Theta(S)$ is $\Delta_{S}$, the diagonal of $S \times S$, which, of course, corresponds to the identity map. The unit, or maximum element, of $\Theta(S)$ is $S \times S$, which corresponds to the constant map. The set of coatoms of $\Theta(S)$ is $\operatorname{ch}(S)=\left\{[\lambda] \in \theta(S): \lambda: S \rightarrow 2\right.$ is a $\mathscr{P}^{\circ}$-surmorphism $\}$ where 2 is the two element chain ([8], p. 724). The $\mathscr{P}^{P}$-surmorphisms onto 2 are called characters and the members of $\operatorname{ch}(S)$ are character congruences. A character is a characteristic function on a proper filter of $S$. For each nonzero element $x \in S$ we define $\left[\lambda_{x}\right]$ by $\lambda_{x}(\uparrow x)=1$ and $\lambda_{x}(S \uparrow x)=0$. Character congruences of this type are called principal.

For each pair $a, b \in S$ with $a<b$ there is a congruence $\left[\tau_{a, b}\right]$ which is minimal with respect to identifying $a$ and $b$, it is given by: $(s, t) \in\left[\tau_{a, b}\right]$ if and only if $s=t$ or $\{s, t\} \supseteqq \downarrow b$ and $s \wedge a=t \wedge a$. The set $K(\Theta(S))$ is obtained by taking all finite sups of congruence $\left[\tau_{a, b}\right]([3]$, p. 1192).

2. Bialgebraic congruence lattices. In deciding whether a coatomically generated, algebraic lattice is bialgebraic (really the concern is whether it is coalgebraic) the coatoms play the crucial role. From ([2], p. 15) we know that a coatomically generated complete lattice is coalgebraic if and only if every coatom is also cocompact. When this result is rephrased for congruence lattices we have

Lemma 2.1. Let $S$ be a semilattice. Then $\Theta(S)$ is bialgebraic if and only if every character congruence on $S$ is cocompact in $\Theta(S)$. 
Three very ordinary semilattices will appear very often in our discussion. We will give two of them now.

EXAMPLE D. Let $D$ be the set $\{0\} \cup\{1 / n: n$ is a positive integer $\}$ equipped with the order it inherits from the real numbers.

Example U. Let $U$ be the dual of $D$. $U$ is isomorphic to the set $\{1-1 / n: n=1,2, \cdots\} \cup\{1\}$ with its usual order.

Although $U$ and $D$ will not be given topologies, their implicit topological properties are what will make them suitable test examples. $D$ is the most elementary of examples of a convergent downward directed set while $U$ is the most elementary example of a convergent upward directed set. Because of this "convergence" $D$ will have a nonprincipal, proper filter and $U$ will have a nonprincipal proper ideal and these sets will keep $D$ and $U$ from having bialgebraic congruence lattices.

Without much difficulty it can be seen that $\Theta(D)$ and $\Theta(U)$ are isomorphic. They both have countably many coatoms, only one of which is not principal.

Lemma 2.2. Neither $\Theta(U)$ nor $\Theta(D)$ is bialgebraic. Consequently, no semilattice having either $U$ or $D$ as a quotient can have a bialgebraic congruence lattice.

Proof. We will do the proof for $D$. Let $[\lambda]$ be the nonprincipal character congruence on $D\left(\lambda^{-1}(0)=0\right.$ and $\left.\lambda^{-1}(1)=D \backslash\{0\}\right)$. If $B$ is any finite set of positive integers then $\cap\left\{\left[\lambda_{1 / n}\right]: n \in B\right\}$ is not contained in $[\lambda]$. However, the intersection of all the principal character congruences is $A_{D}$ (since they separate points). Thus $[\lambda]$ is not cocompact.

If $\varphi: S \rightarrow D$ is a $\mathscr{P}$-surmorphism then $\Theta(D)$ is isomorphic to the filter $\uparrow[\varphi]$. Since any principal filter of a bialgebraic lattice would have to be bialgebriac $\Theta(S)$ cannot be bialgebraic.

The situation illustrated in 2.2 is typical for semilattices whose congruence lattices are not bialgebraic.

Theorem 2.3. Suppose that $S$ is a semilattice. Then $\Theta(S)$ is bialgebraic if and only if $S$ has neither $U$ nor $D$ as a quotient.

Proof. The previous lemma takes care of one half of the proof. Conversely, suppose that $S \in \mathscr{P}$ and $\Theta(S)$ is not bialgebraic. From Lemma 2.1, $S$ will have a noncocompact character congruence-call it 
$[\lambda]$. There must be a downward directed family of congruences $\mathscr{F}=\left\{\left[\psi_{\gamma}\right]: \gamma \in \Gamma\right\}$ such that no member of $\mathscr{F}$ is contained in $[\lambda]$ whereas $\cap \mathscr{F} \supseteqq[\lambda]$. There will be two cases. In one we will get $D$ as a quotient and in the other $U$.

Case 1. Suppose that $[\lambda]$ is a princidal character congruence. Let $e=\inf \lambda^{-1}(1)$. For each $\gamma \in \Gamma$ we define $A_{\gamma}$ to be the filter $\uparrow \psi_{\gamma}^{-1} \circ \psi_{\gamma}(e)$. If for some $\gamma \in \Gamma$ we had $A_{\gamma}=\uparrow e$ then $\psi_{\gamma} \circ \psi_{\gamma}(e) \subseteq \uparrow e=$ $\lambda^{-1} \circ \lambda(e)$ which would lead to the conclusion that $\uparrow e$ is saturated relative to the congruence $\left[\psi_{r}\right]$ (if $(x, y) \in\left[\psi_{r}\right]$ and $x \in \uparrow e$ then $(e, e \wedge y)=$ $(e \wedge x, e \wedge y) \in\left[\psi_{\gamma}\right]$. Then $e \wedge y \in \uparrow e$ which means that $\left.y \in \uparrow e\right)$. Thus ]$\lambda]$ would contain $\left[\psi_{r}\right]$ contrary to our hypothesis. The family $\left\{A_{\gamma}: \gamma \in \Gamma\right\}$ is then downward directed and has $\uparrow e$ as its intersection but no $A_{r}=\uparrow e$.

Set $C_{0}=S$. We then create a subfamily $\left\{C_{1}, C_{2}, \cdots\right\}$ of the $A_{r}$ 's such that $C_{0} \neq C_{1}$ and for each $i<j, C_{j}$ is a proper subset of $C_{i}$. Define $C_{\infty}=\cap\left\{C_{i}: i=0,1,2, \cdots\right\}$. Clearly, $C_{\infty}$ is a filter on $S$ which contains $\uparrow e$. Define a map $\eta: S \rightarrow U$ by $\eta(s)=\sup \left\{1-1 / n: s \in C_{n}\right\}$. Without difficulty $\eta$ can be shown to be a $\mathscr{P}^{\text {-surmorphism. }}$

Case 2. Suppose that $[\lambda]$ is not principal. Then $\lambda^{-1}(1)$ contains an infinite decreasing sequence $a_{1}, a_{2}, \cdots$. Define $\mu: S \rightarrow D$ by $\mu(x)=$

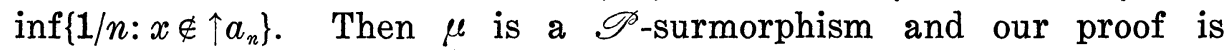
complete.

For some purposes it might be useful to have an internal characterization. This we will given in terms of various sorts of completeness properties. To simplify the statements a bit we introduce a new subcategory of $\mathscr{P}$.

Definition 2.4. Let $\mathscr{W}^{-}$be the subcategory of $\mathscr{P}$ consisting of those semilattices in which every proper filter is principal. The morphisms of $\mathscr{W}$ will be the $\mathscr{P}$-morphisms between objects of $\mathscr{W}$.

Note that membership in $\mathscr{W}^{-}$means that every character congruence is principal. It is also contingent upon the nonexistence of

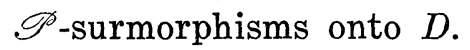

Proposition 2.5. Let $S$ be a semilattice. Then $S \in \mathscr{W}$ if and only if $D$ is not a quotient of $S$.

Proof. If $S \notin \mathscr{W}$ then there is a proper, nonprincipal filter $F$ in $S$. Then as in Case 2 of Theorem 2.3 there is an infinite decreasing sequence $a_{1}>a_{2}>\cdots$ and a $\mathscr{P}^{\text {-surmorphism }} \varphi: S \rightarrow D$ given by $\varphi(x)=\inf \left\{1 / n: x \notin \uparrow a_{n}\right\}$. 
Conversely, if there is a $\mathscr{P}$-surmorphism $\varphi: S \rightarrow D$ then the proper filter $\uparrow \varphi^{-1}(D /\{0\})$ must have a generator if $S$ is to belong to $\mathscr{W}^{-}$. Clearly this is not possible.

As a direct consequence of 2.5 we have

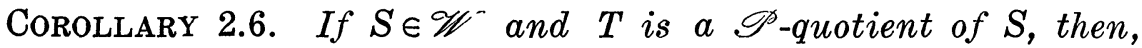
$T$ also belongs to $\mathscr{W}^{-}$.

As further evidence the $\mathscr{P}$-surmorphisms defined on objects of $\mathscr{W}$ are particularly well-behaved, we next show that they are almost residuated.

Proposition 2.7. Suppose that $S$ belongs to $\mathscr{W}$ and $\varphi: S \rightarrow T$ is a $\mathscr{P}$-surmorphism. Define $T^{\prime}$ to be $T$ if $T$ does not have a zero and $T \backslash\left\{0_{T}\right\}$ if $T$ has a zero. Then there is an injective order-preserving map $\psi: T^{\prime} \rightarrow S$ such that $\psi(t)=\inf \phi^{-1}(t)$ and $\psi$ preserves whatever sups exist in $T^{\prime}$.

Proof. Let $t \in T^{\prime}$. Then $\uparrow t$ is a proper filter in $T$. Hence $\uparrow \varphi^{-1}(t)$ is a proper filter of $S$. Then because $S \in \mathscr{W}$, inf $\uparrow \varphi^{-1}(t)$ exists and belongs to $\varphi^{-1}(t)$. Thus $\psi: T^{\prime} \rightarrow S$ is well-defined and clearly orderpreserving.

Now, suppose that $A \subseteq T^{\prime \prime}$ with $\sup A=b$. Then since $\psi: T^{\prime} \rightarrow S$ is order-preserving we have $\psi(b) \geqq \psi(a)$ for all $a \in A$. On the other hand, if $s \in S$ is any other upper bound of $\psi(A)$ then since $\varphi$ is order-preserving, $\varphi(s)$ is an upper bound of $A$ in $T^{\prime}$. Thus $\varphi(s) \geqq b$ and we have $\psi(b) \leqq \psi(\varphi(s)) \leqq s$.

Corollary 2.8. Suppose that $S \in \mathscr{W}$ and $\varphi: S \rightarrow T$ is a $\mathscr{P}$ surmorphism of $S$ onto a chain $T$. Then there is an injective order-preserving map $\psi: T \rightarrow S$ such that $\psi(t)=\inf \varphi^{-1}(t)$ if $t \neq 0_{T}$ and \& preserves arbitrary sups.

Proof. Define $\psi: T^{\prime} \rightarrow S$ as in Proposition 2.7. If $T$ does not have a zero, then we are done. If $S$ and $T$ both have zeros, set $\psi\left(0_{T}\right)=0_{S}$. If $T$ has a zero but $S$ doesn't, then $\uparrow \varphi^{-1}\left(T^{\prime}\right)$ is a proper filter in $S$ and we may let $\psi\left(0_{T}\right)$ be any element of $\downarrow \inf \left(\uparrow \varphi^{-1}\left(T^{\prime}\right)\right) \downarrow$ $\inf \left(\uparrow \varphi^{-1}\left(T^{\prime}\right)\right)$.

An internal characterization of semilattices having bialgebraic congruence lattices is now possible.

THeOREM 2.9. Let $S$ be a semilattice. Then $\Theta(S)$ is bialgebraic 
if and only if $S \in \mathscr{W}^{-}$and it satisfies the following equivalent (in Wु) conditions.

(1) Every bounded ideal in $S$ is principal.

(2) Every element of $S$ is compact.

(3) There is an injective $\mathscr{P}$-morphism from $U$ into $S$.

(4) There is a pair of points $s, t \in S$ with $s<t$ such that the set $\downarrow t \downarrow \downarrow$ contains an infinite chain.

Proof. Using the methods developed in this section conditions (1), (2) and (3) can easily be shown to be equivalent in $\mathscr{W}$ and in that category equivalent to the existence of a $\mathscr{P}$-surmorphism onto $U$. Since membership in $\mathscr{W}$ precludes the existence of a $\mathscr{P}$-surmorphism onto $D$, this result follows from 2.3.

The condition (4) is somewhat different from the others but using the residuation properties of $\mathscr{W}$ it is easily shown to be equivalent to the other three conditions.

In the next section we shall make extensive use of a condition similar to (4) above.

3. Coordinated bialgebraic congruence lattices. Our aim in this section is to characterize those semilattices whose congruence lattices have stronger convergence properties than do bialgebraic lattices. Before we can give the appropriate definition some topology must be discussed.

A topological semilattice (join-semilattice) is a triple $(S, \tau, \wedge)$ $((S, \tau, \vee))$ where $(S, \tau)$ is a Hausdorff topological space and $(S, \wedge)$ is a semilattice $((S, \vee)$ is a join-semilattice) such that $\wedge: S \times S \rightarrow S$ $(\vee: S \times S \rightarrow S)$ is a continuous map when $S \times S$ is equipped with the cartesian product topology. A topological lattice is a quadruple $(L, \tau, \wedge, \vee)$ such that $(L, \tau, \wedge)$ is a topological semilattice and $(L$, $\tau, \vee)$ is a topological join-semilattice. An algebraic lattice $L$ possesses a naturally defined topology, call it $\tau_{\wedge}(L)$, which is generated by declaring $\uparrow k$ to be open and closed for every compact element $k$ of $L$. This topology is compact, Hausdorff and totally disconnected. Moreover, $\left(L, \tau_{\wedge}(L), \wedge\right)$ is a topological semilattice ([5], p. 41). If $L$ is bialgebraic then it will support a second compact, Hausdorff, totally disconnected topology, call it $\tau_{\vee}(L)$, relative to which ( $L$, $\left.\tau_{\vee}(L), \vee\right)$ is a topological join-semilattice. (Of course, $\tau_{\vee}(L)$ is generated by declaring $\downarrow x$ open and closed for each co-compact element $x$ of $L$.) From the continuity of operations in these topologies it follows that both $\tau_{\wedge}(L)$ and $\tau_{\vee}(L)$ contain the interval topology. 
In his dissertation [7] Lystad has proved that a bialgebraic lattice $L$ will support a compact, Hausdorff topology $\tau$ which makes it into a topological lattice if and onjy if $\tau_{\wedge}(L)=\tau_{\wedge}(L)$. In which case, $\tau_{\wedge}(L)=\tau$. With this result as motivation we give the following definition.

Definition 3.1. A bialgebraic lattice $L$ is said to be coordinated if the two intrinsic topologies $\tau_{\wedge}(L)$ and $\tau_{\vee}(L)$ coincide. A bialgebraic lattice that is not coordinated is said to be uncoordinated.

We shall give both an internal and extenal characterization of those semilattices having coordinated bialgebraic congruence lattices. Many of the propositions are concerned with the category $\mathscr{W}$. This is not so surprising in view of the characterizations in the previous section.

At this point our third familiar and elementary lattice will be introduced. It is the prototype of an uncoordinated bialgebraic lattice.

EXAMPLE A. Let $A_{0}$ be an infinite countable set and let 0,1 be two points not in $A_{0}$. Define $A=A_{0} \cup\{0,1\}$ to be the lattice whose partial order is generated by declaring $0<a<1$ for all $a \in A_{0}$ $(<$ means strict inequality here).

While $U$ and $D$ may be thought of as illustrations of convergence on elementary directed sets, Example A will illustrate the problems associated with convergence (or potential convergence) across an anti-chain.

The two topological $\tau_{\vee}(A)$ and $\tau_{\wedge}(A)$ do not coincide. To see this, note that the points of $A_{0}$ are isolated relative to both topologies. Then since 1 is compact and 0 is co-compact, $\{1\} \in \tau_{\wedge}(A)$ and $\{0\} \in \tau_{\vee}(A)$. Thus if $\tau_{\wedge}(A)$ and $\tau_{\vee}(A)$ were to coincide $\tau_{\wedge}(A)$ would be discrete. But as was mentioned before $\left(A, \tau_{\wedge}(A)\right)$ is an infinite compact space! The problem here is that $\bar{A}_{0}=A_{0} \cup\{1\}$ for $\tau_{\vee}(A)$ while $A_{0}=\bar{A}_{0} \cup\{0\}$ for $\tau_{\wedge}(A)$. (In [9] Stepp proves that $\left(A, \tau_{\wedge}(A), \wedge\right)$ cannot be embedded in a compact topological lattice.) For $\Theta(A)$ the situation is similar.

Proposition 3.2. $\Theta(A)$ is an uncoordinated bialgebraic lattice.

Proof. Since $A$ has no infinite chains it has neither $U$ nor $D$ as a quotient. Thus $\Theta(A)$ is bialgebraic. To prove that $\Theta(A)$ is uncoordinated we first show that the congruence $A \times A$ is compact in $\Theta(A)$. Suppose that this is not so. Then there is an upward directed set $\mathscr{C}$ contained in $\Theta(A) \backslash\{A \times A\}$ such that $\sup \mathscr{C}=A \times A$. If there is $[\sigma] \in \mathscr{C}$ such that $\sigma^{-1} \circ \sigma(1)$ contained two distinct elements 
of $A_{0}$, say $a_{1}$ and $a_{2}$, then $(0,1)=\left(a_{1}, 1\right) \wedge\left(a_{2}, 1\right)$ would have to be in $[\sigma]$, which would imply that $[\sigma]=A \times A$. If we always have $\sigma^{-1} \circ \sigma(1)=\{1\}$ then $[\sigma] \subseteq\left[\lambda_{1}\right]$ which would imply that $\left[\lambda_{1}\right] \geqq \sup \mathscr{C}$ while if $a \in \sigma^{-1} \circ \sigma(1)$ for some $[\sigma] \in \mathscr{C}$ then $\sup \mathscr{C} \leqq\left[\lambda_{a}\right]$. We can conclude that $A \times A$ is compact in $\Theta(A)$.

Then $\Theta(A) /\{A \times A\}$ is a closed, and hence compact, subset of $(A) \Theta$ relative to the topology $\tau_{\wedge}(\Theta(A))$. But $\{\downarrow[\lambda]:[\lambda] \times \operatorname{ch}(A)\}$ is an infinite open cover of $\Theta(A) \backslash\{A \times A\}$ which cannot be reduced. Therefore $\Theta(A)$ is not coordinated.

CoROllary 3.3. If $S \in \mathscr{P}$ and $\Theta(S)$ is a coordinated bialgebraic lattice then $S$ cannot have $U, D$ or $A$ as a quotient.

Proof. If $\varphi: S \rightarrow A$ is a $\mathscr{P}$-surmorphism then $\uparrow[\varphi]$ is isomorphic with $\Theta(A)$. If $\Theta(S)$ were a coordinated bialgebraic lattice then so would $\uparrow[\varphi]$, and consequently $\Theta(A)$. This is contrary to Proposition 3.2. An appeal to Theorem 2.3 completes the proof.

In the next lemma we learn a bit more about those semilattices which have $A$ as a quotient. Compare the condition in this lemma with (4) of Theorem 2.9.

LeMma 3.4. Let $S \in \mathscr{W}$ and suppose that $S$ has $A$ as a quotient. Then there is a pair of points $x, y \in S$ such that $x<y$ and $\downarrow y \backslash \downarrow x$ is infinite.

Proof. Suppose that $\varphi: S \rightarrow A$ is a $\mathscr{P}$-surmorphism. From Proposition 2.7 there is an injective order-preserving map $\psi: A /\{0\} \rightarrow S$. Select $x \in \Phi^{-1}(0)$ such that $x<\psi(1)$. Then $\downarrow \psi(1) / \downarrow x$ is an infinite set since it contains $\psi\left(A_{0}\right)$.

With the finiteness condition of the previous lemma in mind we create a new subcategory of $\mathscr{P}$.

Definition 3.5. Let $\mathscr{P}$ be the subcategory of $\mathscr{P}$ consisting of those semilattices $S$ such that

(i) every proper filter of $S$ is principal (i.e., $S \in \mathscr{W}$ );

(ii) if $x, y \in S$ with $x<y$ then $\downarrow y \backslash \downarrow x$ is finite.

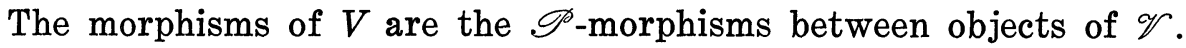

Proposition 3.6. Let $S$ be a semilattice. Then $S \in \mathscr{V}$ if and only if $S$ does not have either $U, D$ or $A$ as a quotient.

Proof. Suppose that $S \in \mathscr{V}$. Then $S \in \mathscr{W}$ and from Proposition 
2.5, $D$ cannot be a quotient of $S$. From Lemma $3.4 A$ cannot be a quotient of $S$. If $U$ were a quotient of $S$ then from Proposition 2.7 $S$ would have to contain a copy of $U$ which would violate the second condition for membership in $\mathscr{V}$.

Now suppose that none of the test semilattices is a quotient of $S$. Then from Proposition $2.5 S$ belongs to $\mathscr{W}$ so we need only be concerned with the second condition of Definition 3.5. Let $s, t \in S$ with $s<t$. From Theorem 2.9 every chain in $\downarrow t \backslash \downarrow s$ is finite. Suppose that $\downarrow t \downarrow \downarrow s$ is infinite. Form a chain $t=x_{1}>x_{2}>x_{3}$ where $x_{i}$ covers $x_{i+1}$ and $\downarrow x_{i} / \downarrow s$ is infinite. Since every chain in $\downarrow t \mid \downarrow s$ is finite there is $x_{j} \in \downarrow t \mid \downarrow s$ such that $\downarrow x_{j} \backslash \downarrow s$ is infinite but for each $x \in \downarrow x_{j} \mid$ $\left(\downarrow s \cup\left\{x_{j}\right\}\right), \downarrow x \backslash \downarrow s$ is finite. It follows that $x_{j}$ must cover a countably infinite subset $C$ in $\downarrow x_{j} \mid \downarrow s$. Let $\beta$ : $C \rightarrow A_{0}$ be a bijection. Then define a map $\varphi: S \rightarrow A$ by

$$
\varphi(s)=\left\{\begin{array}{cl}
1 & \text { if } s \in \uparrow x_{j} \\
\beta\left(x_{j} \wedge s\right) & \text { if } x_{j} \wedge s \in C \\
0 & \text { otherwise }
\end{array}\right.
$$

Then $\varphi$ will be a $\mathscr{P}$-surmorphism which contradicts the assumption that $\downarrow t \backslash \downarrow s$ was infinite.

Lemma 3.7. Suppose that $S \in \mathscr{Y}$.

(1) If $\varphi: S \rightarrow T$ is a $\mathscr{P}$-surmorphism, then $T$ also belongs to $\mathscr{V}$.

(2) If $x \in S$, then there is a finite set $B$ such that $\downarrow x \mid\{x\}=\downarrow B$.

Proof. (1) follows from Proposition 3.6. (2) Let $y \in \downarrow x \mid\{x\}$. Then since $\downarrow x \mid \downarrow y$ is finite it follows that $\downarrow x \backslash\{x\}=\downarrow((\downarrow x \backslash \downarrow y) \cup\{y\})$.

Proposition 3.8. Let $S \in \mathscr{V}$. Then $\Theta(S)$ is a coordinated bialgebraic lattice.

Proof. We need only show that $\tau_{\wedge}=\tau_{\wedge}(\Theta(S))$ coincides with $\tau_{\vee}=\tau_{\mathrm{v}}(\Theta(S))$. Since $S \in \mathscr{P}$ every character congruence is of the form $\left[\lambda_{a}\right]$. Then since $\Theta(S)$ is coatomically generated we see that $\tau_{V}$ is generated by the sets $\downarrow\left[\lambda_{a}\right]$. We shall show that $\downarrow\left[\lambda_{a}\right]$ belongs to $\tau_{\wedge}$. Let $C$ be the finite set of maximal elements in $\downarrow a \backslash\{a\}$. We claim that $\Theta(S)$ is the disjoint union of $\downarrow\left[\lambda_{a}\right]$ and $B=\cup\left\{\uparrow\left[\tau_{a, c}\right]\right.$ : $c \in C\}$. These two sets are disjoint since to gain membership in $\downarrow\left[\lambda_{a}\right]$ a congruence $[\varphi]$ must have $\varphi^{-1} \circ \varphi(a) \cong \uparrow a$, while to belong to $B, \varphi^{-1} \circ \varphi(a) \cap S \backslash \uparrow a$ must be nonempty. Now suppose that $[\varphi] \in \Theta(S)$. If $\varphi^{-1} \circ \varphi(\alpha) \cong \uparrow a$ then $[\varphi] \in \downarrow\left[\lambda_{a}\right]$ whereas if there is $s \in S \backslash \uparrow a$ such that $(s, a) \in[\varphi]$ we then have $(\alpha \wedge s, a) \in[\varphi]$. There is $c \in C$ such that $a \wedge s \leqq c<a$. Consequently, $(c, a) \in[\varphi]$ from which it follows that 
$[\varnothing] \in \uparrow\left[\tau_{a, c}\right]$. The set $B$ is a finite union of open and closed sets relative to $\tau_{\wedge}$ therefore $\downarrow\left[\lambda_{a}\right]$ is also open and closed with respect to $\tau_{\wedge}$.

The topology $\tau_{\wedge}$ is generated by declaring the sets $\uparrow\left[\tau_{a, b}\right]$ to be open and closed for $a<b$ in $S$. The set $R=\downarrow b \downarrow_{\downarrow} a$ is finite since $S \in \mathscr{V}$. We claim that $\Theta(S)$ is a disjoint union of $\uparrow\left[\tau_{a, b}\right]$ aud $\cup\left\{\downarrow\left[\lambda_{r}\right]: r \in R\right\}$. The proof is very similar to that given above so we shall omit it. We have therefore shown that $\tau_{\wedge}=\tau_{\vee}$. Thus $S$ is a coordinated bialgebraic lattice.

We are now ready for our characterization.

THEOREM 3.9. Let $S \in \mathscr{P}$. Then the following three conditions are equivalent:

(1) $\Theta(S)$ is a coordinated bialgebraic lattice.

(2) $S$ does not have either $U, D$ or $A$ as a quotient.

(3) $S$ belongs to $\mathscr{V}$.

Proof. This result follows from Corollary 3.3, Proposition 3.6 and Proposition 3.8.

Let $\mathscr{A}$ be the category of algebraic lattices. The morphisms of $\mathscr{A}$ will be maps between $\mathscr{A}$-objects which preserve arbitrary infs and sups of upward directed sets. This category with slight modification was discussed extensively in [5]. The particular morphisms of $\mathscr{A}$ were needed to establish an equivalence of categories in that paper. In the remainder of this section we will be concerned with finding those semilattices whose congruence lattices are Boolean algebras or $\mathscr{A}$-quotients of Boolean algebras and with narrowing down the family of coordinated lattices which could be congruence lattices.

If $\Theta(S)$ is a Boolean algebra for some semilattice $S$ then since $\Theta(S)$ is complete and coatomic by Tarski's theorem it must be isomorphic to $2^{B}$, the power set of some set $B$. The power set Boolean algebras are easily seen to be coordinated bialgebraic lattices.

Proposition 3.10. Let $S \in \mathscr{P}$. Then $\Theta(S)$ is a Boolean algebra if and only if $S$ has neither $U, D, A$ or $2^{2}$ as a quotient, in which case $S$ is isomorphic with $2^{\operatorname{ch}(S)}$.

Proof. From 3.9 we know that $\Theta(S)$ will be a coordinated bialgebraic lattice if and only if $S$ does not have $U, D$ or $A$ as a quotient. From ([3], p. 1195) or ([8], p. 727) we know that $\Theta(S)$ is distributive if and only if no two noncomparable elements of $S$ have a common upper bound. This condition is equivalent to $S$ not hav- 
ing $2^{2}$ as a quotient. To complete the first part of the proof we need only note that a coatomically generated, distributive, coalgebraic lattice is a Boolean algebra ([2], p. 30).

The second part of this proposition follows from the fact that $\operatorname{ch}(S)$ is the set of coatoms of $\Theta(S)$.

In the last result we could replace $2^{\operatorname{ch}(S)}$ by $2^{S^{\prime}}$, where $S^{\prime}$ is $S$ if $S$ does not have a zero and $S \backslash\left\{0_{S}\right\}$ otherwise, since $S$ belongs to $\mathscr{W}$ and every character congruence would then be principal! Compare Proposition 3.10 with Theorem 5 of [11] and Theorem 4.10 of [4]. We rephrase Corollary 10 of [10] as follows:

THEOREM 3.11. If $S$ is a coatomically generated algebraic lattice, then it is an $\mathscr{A}$-quotient of a power set lattice if and only if for each $k \in K(S), \uparrow k$ contains all but finitely many coatoms of $S$.

In our last theorem we shall greatly reduce the number of coordinated bialgebraic lattices which are potential congruence lattices for semilattices. In so doing we obtain another characterization (of sorts) of coordinated bialgebraic congruence lattices. Again, the lattice $A$ plays an important role.

LeMma 3.12. Suppose that $S$ is a semilattice for which $\Theta(S)$ is not bialgebraic, then $\Theta(S)$ is not an $\mathscr{A}$-quotient of a power set.

Proof. From 2.3 we know that $S$ must have either $U$ or $D$ as a quotient. Suppose that there is a $\mathscr{P}$-surmorphism $\varphi: S \rightarrow U$. (The following proof can be easily modified to take care of the case in which $D$ is a quotient of $S$.) The minimum congruence identifying $1 / 2$ and $1,\left[\tau_{1,1 / 2}\right]$ belongs to $K(\Theta(U))$. However $\left[\tau_{1,1 / 2}\right]=\left[\lambda_{1 / 2}\right]$. Thus $\uparrow\left[\tau_{1,1 / 2}\right]=\left\{\left[\tau_{1,1 / 2}\right], U \times U\right\}$. Then since $\Theta(U)$ has an infinite number of coatoms we see from Theorem 3.11 that $\Theta(U)$ cannot be an $\mathscr{A}$ quotient of a power set.

Now, suppose that there is an $\mathscr{A}$-surmorphism $\delta: 2^{B} \rightarrow \Theta(S)$. The set $\delta^{-1}(\uparrow[\varphi])$ is a principal filter of $2^{B}$. Thus it is an $\mathscr{A}$-quotient of $2^{B}$. On the other hand $\uparrow[\varphi]$ is isomorphic to $\Theta(U)$. We would then have $\Theta(U)$ an $\mathscr{A}$-quotient of a power set contrary to the previous paragraph. Therefore $S$ is not the $\mathscr{A}$-quotient of a power set.

Lemma 3.13. The lattice $A$ is an $\mathscr{A}$-quotient of $\Theta(A)$.

Proof. We define a map $\zeta: \Theta(A) \rightarrow A$ by setting $\zeta(A \times A)=1$, 
$\zeta\left(\left[\lambda_{a}\right]\right)=a$ for $a \in A_{0}$, and $\zeta([\sigma])=0$ otherwise. This map will be an $\mathscr{A}$-surmorphism because $\zeta^{-1}(0)=\downarrow\left[\lambda_{1}\right]$.

THEOREM 3.14. Let $S$ be a semilattice. Then $\Theta(S)$ is a coordinated bialgebraic lattice if and only if it is an $\mathscr{A}$-quotient of a power set.

Proof. From Lemma 3.12 we can restrict our attention to those semilattices $S$ for which $\Theta(S)$ is bialgebraic. Suppose that $\Theta(S)$ is a coordinated bialgebraic lattice and suppose that $a, b \in S$ with $a<b$. Then relative to the topology $\tau_{\wedge}(\Theta(S)), \uparrow\left[\tau_{a, b}\right]$ is an open and closed neighborhood of the congruence $S \times S$. Since $\Theta(S)$ is coatomically generated $\Theta(S) \backslash \uparrow\left[\tau_{a, b}\right]=\cup\left\{\downarrow\left[\lambda_{x}\right]: x \in E\right\}$ for some subset $E$ of $S$. (Recall that $S \in \mathscr{W}$ every character congruence is principal.) Each $\downarrow\left[\lambda_{x}\right]$ is open and closed in $\tau_{\vee}(\Theta(S))$. But $\Theta(S)$ is coordinated so they are open and closed in $\tau_{\wedge}(\Theta(S))$. However, the family $\left\{\downarrow\left[\lambda_{x}\right]: x \in E\right\}$ is a nonreducible open cover of the compact set $\Theta(S) \backslash \uparrow\left[\tau_{a, b}\right]$. Hence $E$ must be finite. Then by Theorem $3.11, \Theta(S)$ is an $\mathscr{A}$-quotient of a power set.

Conversely, suppose that there is an $\mathscr{A}$-surmorphism $\varphi: 2^{B} \rightarrow$ $\Theta(S)$ for some set $B$. If $\Theta(S)$ were not coordinated then there

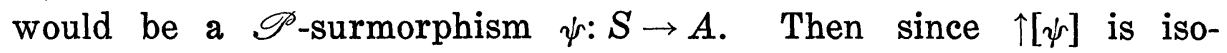
morphic to $\Theta(A)$ and $\varphi^{-1}\left(\uparrow[\psi]\right.$ ) is a principal filter of $2^{B}$ (and hence an $\mathscr{A}$-quotient of $2^{B}$ ) it follows that $\Theta(A)$ is an $\mathscr{A}$-quotient of $2^{B}$. But from Lemma 3.13 we know that $A$ is an $\mathscr{A}$-quotient of $\Theta(A)$. Hence $A$ is an -quotient of $2^{B}$ contradicting Theorem 3.11 .

\section{REFERENCES}

1. C. R. Atherton, Concerning intrinic topologies on Boolean algebras and certain bicompactly generated lattices, Glasgow Math. J., 11 (1970), 156-161.

2. P. Crawley and P. R. Dilworth, The Algebraic Theory of Lattices, Prentice Hall, 1973.

3. R. A. Dean and R. H. Oehmke, Idempotent semigroups with distributive right congruence lattices, Pacific J. Math., 14 (1964), 1187-1209.

4. S. Fajtlowicz and S. Schmidt, Bézout families, join-congruences and meet-irreducible ideals, Colloq. Math. Soc. Jànos Bolyai, 14 (1974), 51-76.

5. K. H. Hofmann, M. Mislove and A. Stralka, The Pontryagin Duality of Compact O-dimensional Semilattices and its Applications, Lecture Notes in Math. 396, SpringerVerlag, Heidelberg, 1974.

6. D. C. Kent, and C. R. Atherton, The order-topology in a bicompactly generated lattice, J. Australian Math. Soc., 8 (1968), 345-349.

7. G. S. Lystad, Dissertation, University of California, Riverside, 1978.

8. Dona Papert Strauss, Congruence relations in semilattices, J. London Math. Soc., 39 (1964), 723-729.

9. J. W. Stepp, Semilattices which are embeddable topological lattices, J. London Math. Soc., (2) 7 (1973), 76-82. 
10. A. Stralka, Quotients of products of compact chains, Bull. London Math. Soc., 11 (1979), 1-4.

11. J. Varlet, Congruences dans les demi-lattis, Bull. Soc. Roy, Soc. Liege, 34 (1965), 231-240.

Received January 28, 1978 and in revised form September 13, 1978.

UNIVERSITY OF CALIFORNIA

RIVERSIDE, CA 92521 



\section{PACIFIC JOURNAL OF MATHEMATICS}

\section{EDITORS}

DONALD BABBITT (Managing Editor)

University of California

Los Angeles, California 90024

HUgo RossI

University of Utah

Salt Lake City, UT 84112

C. C. MOORE and ANDREW OGG

University of California

Berkeley, CA 94720

\section{J. DUGUNDJI}

Department of Mathematics University of Southern California Los Angeles, California 90007

R. Finn and J. Milgram Stanford University Stanford, California 94305

\section{ASSOCIATE EDITORS}

E. F. BECKENBACH

B. H. NEUMANN

F. WOLF

K. YoshidA

\section{SUPPORTING INSTITUTIONS}

UNIVERSITY OF BRITISH COLUMBIA CALIFORNIA INSTITUTE OF TECHNOLOGY UNIVERSITY OF CALIFORNIA MONTANA STATE UNIVERSITY UNIVERSITY OF NEVADA, RENO NEW MEXICO STATE UNIVERSITY OREGON STATE UNIVERSITY UNIVERSITY OF OREGON
UNIVERSITY OF SOUTHERN CALIFORNIA STANFORD UNIVERSITY UNIVERSITY OF HAWAII UNIVERSITY OF TOKYO UNIVERSITY OF UTAH WASHINGTON STATE UNIVERSITY UNIVERSITY OF WASHINGTON 


\section{Pacific Journal of Mathematics}

Vol. 85, No. $1 \quad$ September, 1979

Ralph Alexander, Metric averaging in Euclidean and Hilbert spaces...... 1

B. Aupetit, Une généralisation du théorème de Gleason-Kahane-Żelazko

pour les algèbres de Banach..............................

Lung O. Chung, Jiang Luh and Anthony N. Richoux, Derivations and

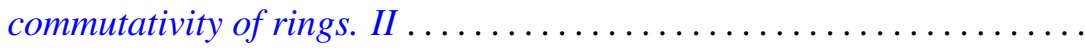

Lynn Harry Erbe, Integral comparison theorems for third order linear

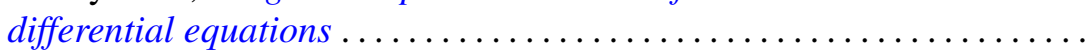

Robert William Gilmer, Jr. and Raymond Heitmann, The group of units of a

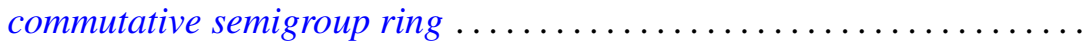

George Grätzer, Craig Robert Platt and George William Sands, Embedding lattices into lattices of ideals ...........................

Raymond D. Holmes and Anthony Charles Thompson, $n$-dimensional area and content in Minkowski spaces ....................... 77

Harvey Bayard Keynes and M. Sears, Modelling expansion in real flows....

Taw Pin Lim, Some classes of rings with involution satisfying the standard

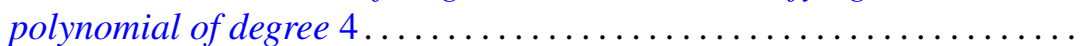

Garr S. Lystad and Albert Robert Stralka, Semilattices having bialgebraic congruence lattices ................................... 131

Theodore Mitchell, Invariant means and analytic actions . . . . . . . . . 145

Daniel M. Oberlin, Translation-invariant operators of weak type ........ 155

Raymond Moos Redheffer and Wolfgang V. Walter, Inequalities involving

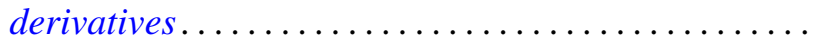

Eric Schechter, Stability conditions for nonlinear products and semigroups ................................

Jan Søreng, Symmetric shift registers ........................ 201

Toshiji Terada, On spaces whose Stone-Čech compactification is $\mathrm{Oz} \ldots \ldots .231$

Richard Vrem, Harmonic analysis on compact hypergroups ... 